\title{
Assessing GastroPanel serum markers as a non-invasive method for the diagnosis of atrophic gastritis and Helicobacter pylori infection
}

\author{
Dominique Noah Noah ${ }^{1 *}$, Marie Claire Okomo Assoumou ${ }^{2}$, Servais Albert Fiacre Eloumou Bagnaka ${ }^{3}$, \\ Guy Pascal Ngaba ${ }^{4}$, Ivo Ebule Alonge ${ }^{2}$, Lea Paloheimo ${ }^{5}$, Oudou Njoya ${ }^{2,6}$ \\ ${ }^{1}$ Yaounde Central Hospital, Faculty of Medicine and Pharmaceuticals Sciences, University of Douala, Douala, Cameroon \\ ${ }^{2}$ Faculty of Medicine and Biomedical Sciences, University of Yaounde, Yaounde, Cameroon \\ ${ }^{3}$ Douala General Hospital, Faculty of Medicine and Pharmaceuticals Sciences, University of Douala, Douala, Cameroon \\ ${ }^{4}$ District Hospital of Bonassama, Faculty of Medicine and Pharmaceuticals Sciences, University of Douala, Douala, Cameroon \\ ${ }^{5}$ Biohit, Helsinki, Finland \\ ${ }^{6}$ University Teaching Hospital, Yaounde, Cameroon \\ Email: "noahnoahd@yahoo.fr
}

Received 10 May 2012; revised 11 June 2012; accepted 25 June 2012

\section{ABSTRACT}

Gastric colonization by Helicobacter pylori increases the risk of gastric disorders, including atrophic gastritis which can be diagnosed based on levels of serum biomarkers like Gastrin and Pepsinogen. We therefore examined the efficacy of a serological-based method namely GastroPanel Blood kit, in diagnosing and scoring gastritis associated to Helicobacter pylori infection. Patients with dyspeptic symptoms were prospectively recruited on voluntary basis at the Yaounde Central Hospital and University Teaching Hospital, from March to July 2011. The degree of atrophy was classified according to levels in patient serum of pepsinogens I and II (PGI and PGII) and Gastrin 17 (G17) and compared with histological profiles as reference method. A specific ELISA test was used for the detection of $\boldsymbol{H}$. pylori IgG antibodies. In total, 86 volunteers from 21 to 83 years old $($ mean $=46.4 \pm 3.3$ ) were enrolled, including $74.4 \%$ of women and $25.6 \%$ of men. The prevalence of gastritis was statistically similar between Gastro Blood Panel test and histology used as reference method $(89.5 \%$ versus $83.7 \%$ : $p>0.20)$. Diagnosis based on serum makers showed high sensitivity $(\mathbf{9 3 . 1 \% )}$ in comparison with the reference method. However, the serological based method has diagnosed more atrophic gastritis than the reference (17.4\% versus $7.0 \%$ : p $<0.01$ ), especially at antrum of stomach with $H$. pylori infection. The prevalence of $H$. pylori infection was $81.4 \%$ with histology versus $84.9 \%$ with serology (GBP) (p > 0.05). Furthermore, the prevalence of $H$. pylori infection did not differ significantly between serological method $(84.9 \%)$ and reference

${ }^{*}$ Corresponding author. method (81.4\%). These results suggest that diagnosis of atrophic gastritis and $H$. pylori infection obtained with an optional serological method (GastroPanel) is in a strong agreement with the biopsy findings, and thus can be a useful non endoscopic assessment of stomach mucosal atrophy in patients with dyspepsia.

Keywords: Diagnosis; Atrophic Gastritis; Helicobacter pylori; Pepsinogen; Gastrin

\section{INTRODUCTION}

Infection of the gastric mucosa by Helicobacter pylori and Atrophic gastritis increase the risk of various gastric disorders, including peptic ulcers and gastric cancer. The usual cause of dyspepsia is gastritis, which is generally diagnosed by histological analysis of a gastric biopsy taken during endoscopy. Gastric biopsies performed in both the antrum and corpus have crucial role for establishing the the agent responsible for the injury, the pattern of gastritis (e.g. non atrophic, atrophic mucosa or intestinal metaplasia) [1]. Serum levels of gastrin-17 and pepsinogen I are well known biomarkers of atrophic gastritis of antrum and corpus respectively. PGI is secreted exclusively by the chief and mucous neck cells of the corpus and fundus and reflect the histological status of the corpus of the stomach. G-17 is the antral hormone produced by the the G-cells of the mucosa of the antrum and regulates gastric acid secretion and growth of the gastric mucosa. G-17 will be depressed in cases of atrophy in the stomach [2-6]. Previously, the only way to get reliable information about the stomach mucosa had been gastroscopy and histological analysis of the biopsy. Now, newer methods are continually being developed and 
investigated. Using the blood test GastroPanel developed by Biohit plc, Helsinki-Finland, quick screening and diagnosis of $H$. pylori infection and atrophic gastritis, as well as evaluation of risk factors of gastric cancer and peptic ulcer disease has been achieved [7]. Many studies have been reported on the evaluation of blood tests to predict normal gastric mucosa and screening markers for chronic atrophic gastritis. The efficacy of the panel is evaluated in terms of the specificity and sensitivity and its correlation with the endoscope and whether or not endoscopic examination can be avoided if the GastroPanel indicates healthy and normal. Thus our study was aimed at demonstrating the usefulness of the GastroPanel test in the diagnosis of $H$. pylori infection and atrophic gastritis in Cameroon. In this paper, the levels of $H$. pylori antibodies and pepsinogen and gastrin markers are combined to propose an optional decision tree based on a serological kit (GastroPanel test) for the diagnosis of $H$. pylori infection and dyspeptic symptoms in patients.

\section{MATERIAL AND METHODS}

\subsection{Patient Information}

The study was carried out from March to July 2011, at the Yaounde Central Hospital (YCH) and University Teaching Hospital (UTH). Patients were recruited prospectively on voluntary basis according to the following criteria: Adult patients (over 20 years) and undergo endoscopy with biopsy for dyspeptic symptoms. Patients: in whom endoscopy is carried out for other than diagnostic reasons, or as an "emergency examination" due to bleeding etc., was excluded from the study. Patients fasted for 10 hours before sample collection and were exemted medication which had effects on gastric acid secretion such as aluminium hydroxide containing drugs or gel, bismuth, sodium alginate, sodium bicarbonate, magnesium hydroxide gel, magnesium trisilicate mixture, magnesium carbonate, and calcium carbonate were avoided.

\subsection{Blood Samples}

Basal blood was drawn into EDTA tubes from each patient and was instantly centrifuged at $2000 \mathrm{~g}$ for $15 \mathrm{~min}-$ utes for plasma collection. Plasma samples were then distributed into cryovials and stored at $-20^{\circ} \mathrm{C}$ until they were tested. The commercial kit GastroPanel (Biohit plc Helsinki, Finland) served to determine serological levels of PGI, PGII, G17 and $H$. pylori IgG antibodies. Specific enzyme immnuno assays (ELISA) were performed on micro well plates according to instructions of the manufacturer for the measurement of the absorbance after a peroxidation reaction at $450 \mathrm{~nm}$. Linear graphs on standard concentrations were used to estimate unknown sample concentrations and the $H$. pylori Abs were expressed as enzyme immune units (EIU). Any blood sample with EIU $\geq 30$ was considered as positive for $H$. pylori antibodies. For the diagnosis of gastritis, any sample with EIU $<30$ (negative for $H$. pylori IgG) and ratio PGI/PGII $>2.5$ (or PGI $\geq 25 \mu \mathrm{g} / \mathrm{l}$ ) and G17 $\geq 1 \mathrm{pmol} / \mathrm{l}$ was considered as normal mucosa (no gastritis); any sample with EIU $\geq 30$ (positive for $H$. pylori IgG) + ratio PGI/PGII > 2.5 (or PGI $\geq 25 \mu \mathrm{g} / \mathrm{l}$ ) if $0.1 \mathrm{pmol} / 1<\mathrm{G} 17<5 \mathrm{pmol} / 1$ was considered as non atrophic (superficial) gastritis, and low levels of G17 ( $\leq 0.05 \mathrm{pmol} / \mathrm{l})$ indicated atrophy at antrum regardless the level of other makers (H.pylori IgG Abs and pepsinogen), while low PGI/PGII ratio $(<2.5)$ or PGI level $(<25 \mu \mathrm{g} / \mathrm{l})$ associated to G17 levels $>0.05$ pmol/1 and $H p$ IgG Abs $\geq 30$ EIU suggested atrophy at corpus. Samples with low levels of PGI/PGII ratio $(<2.5)$ or PGI level $(<25 \mu \mathrm{g} / \mathrm{l})$ associated to low levels of G17 $(\leq 0.05 \mathrm{pmol} / \mathrm{l})$ and $\mathrm{Hp}$ IgG Abs $\geq 30$ EIU were considered as atrophic at both antrum and corpus (Figure 1). The accuracy of the serological commercial kit (Blood Panel Test) in diagnosing gastritis and H. pylori infection was evaluated with reference to endoscopy and biopsy examination [8].

\subsection{Histology}

Routine gastroscopic examinations were complemented by two standard biopsies from the antrum and corpus and set into formalin tubes. The biopsies were processed into paraffin blocks and histological sections were obtained using ordinary techniques and stains (Haematoxillin and Eosin, modified Giemsa (for $\mathrm{H}$. pylori) at the routine histopathology laboratory of the YCH. Experienced pathologist studied the slides by using the Updated Sydney System as criteria in evaluation and grading of mucosal alterations.

\subsection{Statistical Analysis}

The statistics, significance, prevalence, and mean values were calculated by means of the computer program SPSS 12.0 version. All calculations were realized at $95 \%$ confidence interval. Assay analyses were done using the GastroSoft, which is based on an algorithm used to classify gastritis as antral atrophic gastritis, corpus atrophic gastritis, and superficial gastritis or normal stomach. For the accuracy of commercial kit test, the following parameters were estimated: sensitivity (the proportion of patients with atrophic gastritis confirmed as positive by the serological test), specificity as the proportion of patients free for atrophic gastritis confirmed as negative by the test), Positive Predictive Value or PPV as the percentage of patients expected to have gastritis confirmed by a positive test outcome) and Negative Predictive Value or NPV as the percentage of patients expected to be free for gastritis with a negative test outcome). The study was approved by both 


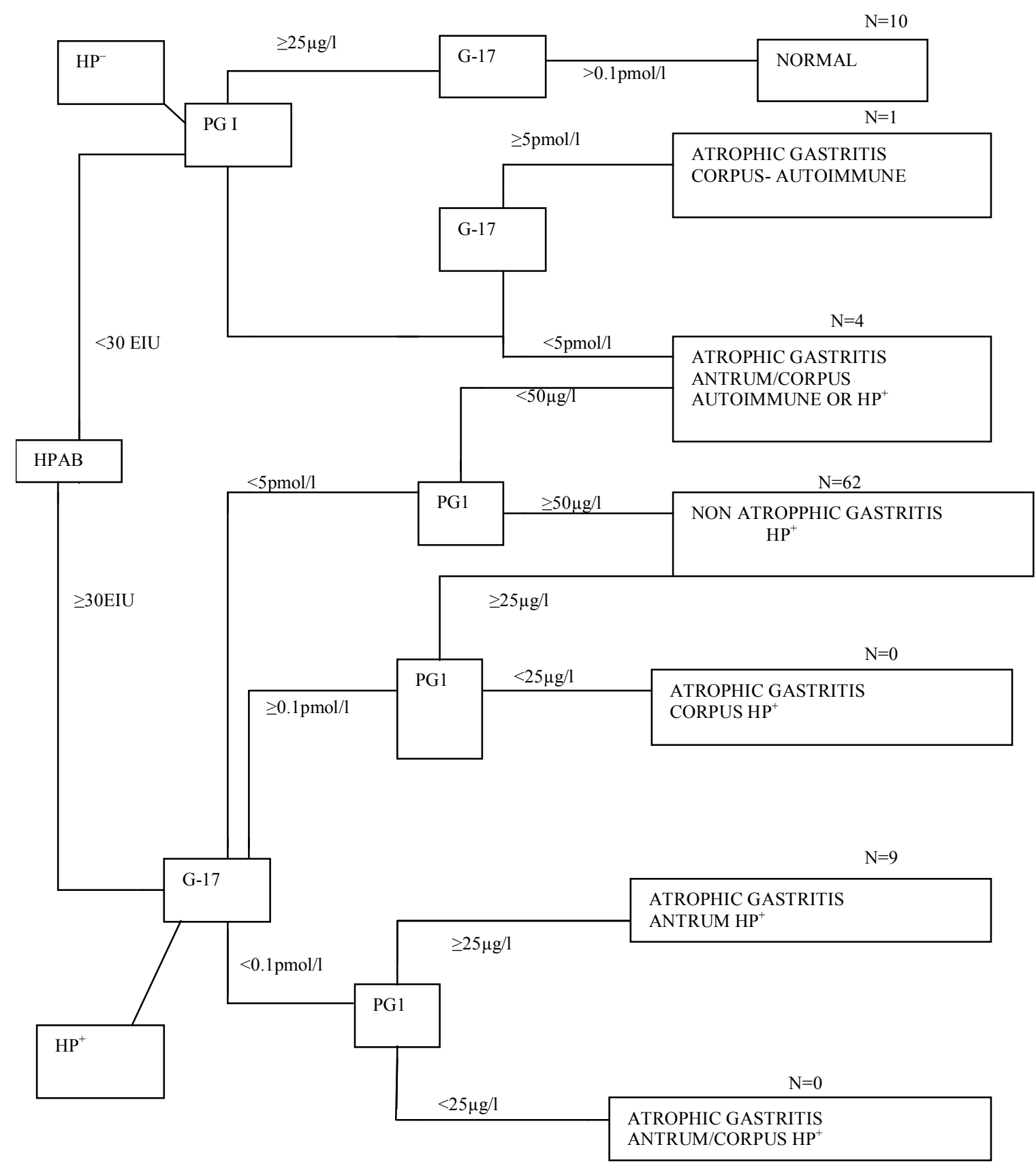

PGI: Pepsinogen I; PG II: Pepsinogen II; G-17: Gastrin -17 fasting levels; HpAB: Helicobacter pylori antibodies-IgG; Hp $^{+}$: Helicobacter pylori positive; $\operatorname{IgG}>30 \mathrm{EIU} ; \mathrm{Hp}^{-}$: Helicobacter pylori negative $\mathrm{IgG}<30 \mathrm{EIU}$.

Figure 1. Algorithm (decision tree) for classification of patients into different categories of atrophic gastritis by the Helicobacter pylori antibody titre (HpAb), and serum levels of PG1 andG-17fast. The absence of evidence of Helicobacter pylori-infection is considered to indicate an autoimmune origin of gastritis. The number of cases in each column arm is indicated.

local ethics committees of the YCH and the UTH. A formal clearance was obtained from the national ethics committee under the authorization Number 065/CNE/SE/2011 of the 17th of March 2011. All information related to patient health and laboratory results were handled confidentially. All patients signed the informed consent form and were informed about their results when the study was completed.

\section{RESULTS}

\subsection{Patient Informations}

The total number of enrolled patients was 86 , including 64 women $(74.4 \%)$ and 22 men (25.6\%), and patient age ranged between 21 and 83 years old with a mean $( \pm$ C.I.) of $46.4( \pm 3.3)$ years old. The study sample comprised 37 
(43\%) patients from 21 to 39 years old, $32(37.2 \%)$ from 40 to 59 years old and $17(19.8 \%)$ patients of 60 years old and above. In patient serum, the mean concentration $( \pm$ C.I.) of serological markers in diagnosis of gastritis or $H$. pylori infection was $122.8 \pm 14.1 \mu \mathrm{g} / \mathrm{l}$ for PGI (from 45 to $337 \mu \mathrm{g} / \mathrm{l}$ ), $25.5 \pm 3.2 \mu \mathrm{g} / \mathrm{l}$ for PGII (from 1.5 to $70 \mu \mathrm{g} / \mathrm{l}$ ), $2.2 \pm 1.6 \mathrm{pmol} / 1$ for $\mathrm{G} 17$ (from 0.04 to $70 \mathrm{pmol} / \mathrm{l}$ ) and $217.9 \pm 57.5 \mathrm{EIU}$ for $\mathrm{Hp}$ IgG Ab (from 12 to $1584 \mathrm{EIU}$ ).

An algorithm based on levels of PGI/PGII ratio, PGI, Gastrin 17 and $H$. pylori IgG antibodies was used for diagnosing gastritis and grading atrophy in blood samples (Figure 1). The prevalence of gastritis among patients consulting for gastric disorders was 76/86 (88.4\%) with serological test. This prevalence did not differ significantly from the prevalence observed $72 / 86(83.7 \%)$ with standard method $(p>0.20)$. Gastritis were classified into two categories including superficial gastritis as non atrophic gastritis 62/86 (72.1\%) with GastroPanel versus 65/86 $(75.6 \%)$ with histology $(\mathrm{p}>0.20)$ and atrophic gastritis 14/86 (16.3\%) with GastroPanel versus 7/86 (8.1\%) with histology $(\mathrm{p}<0.01)$ (Table 1).

\subsection{Accuracy of Blood GastroPanel Kit for the Diagnosis of Gastritis and Atrophy}

Atrophy was then ranked according to the location of inflammation at corpus, at antrum or at both corpus and antrum. The analysis of serological profiles of atrophic gastritis indicated 9/14 (64.3\%) atrophy at antrum, 1/14 $(07.1 \%)$ at corpus and 4/14 (28.6\%) at both corpus and atrum. In parallel with histology, atrophy was diagnosed at $4 / 7$ antrum $(57.1 \%)$, at corpus $1 / 7(14.3 \%)$ and at both corpus and atrum $2 / 7(28.6 \%)$. These results on the distribution of atrophy in stomach suggest a good accuracy of serological markers compared to the routine method. In addition, the sensitivity and specificity of GastroPanel kit for the diagnosis of gastritis in stomach mucosa were $91.7 \%$ and $28.6 \%$ respectively, whereas positive predictive value (PPV) and negative predictive value (NPV) were respectively $87.0 \%$ and $44.4 \%$. For the scoring of atrophy, the sensitivity and specificity of serological test were respectively $85.7 \%$ and $88.6 \%$ with $40.0 \%$ of PPV and $98.6 \%$ of NPV. Decreased levels of PGI $(p=0.003)$ and a PGI/PGII ratio was observed with progression to corpus atrophy. Similarly decreased levels of G-17 were observed with progression to antrum atrophy (Tables 1 and 2).

\subsection{Accuracy of Blood GastroPanel Kit for the Diagnosis of Helicobacter pylori Infection}

High levels of $H$. pylori IgG antibodies ( $\geq 30$ EIU) referred as $H$. pylori infection were recorded among 73/86 $(84.9 \%)$ of patients with serological test. This serological pattern of $H$. pylori infection was statistically similar to the histological pattern that showed 70/86 (81.4\%) of $H$. pylori infection among patients $(\mathrm{p}>0.50)$ (Table 1). The sensitivity and specificity of serological test in the detection of $H$. pylori infection were $95.7 \%$ and $62.5 \%$ respectively, with $91.8 \%$ of PPV and $76.9 \%$ of NPV.

Table 1. Classification of gastritis; GastroPanel markers versus histology.

\begin{tabular}{|c|c|c|c|}
\hline & $\begin{array}{c}\text { Atrophic gastritis } \\
\text { PGI }<25 \mu \mathrm{g} / 1 \text { or } \mathrm{PGI} / \mathrm{PGII}<2.5\end{array}$ & $\begin{array}{c}\text { No atrophy } \\
\text { PGII } \geq 25 \mu \mathrm{g} / 1 \text { or } \mathrm{PGI} / \mathrm{PGII} \geq 2.5\end{array}$ & All cases \\
\hline \multicolumn{4}{|l|}{ Patients } \\
\hline Women & 12 & 52 & 64 \\
\hline Men & 2 & 20 & 22 \\
\hline All samples & 14 & 72 & 86 \\
\hline Mean age $( \pm \mathrm{CI})$ & $47.3( \pm 10.2)$ & $46.5( \pm 3.5)$ & $46.9( \pm 3.3)$ \\
\hline \multicolumn{4}{|l|}{ GastroPanel Markers } \\
\hline Pepsinogen $\mathrm{I} / \mu \mathrm{g} / 1( \pm \mathrm{CI})$ & $61.7( \pm 14.3)$ & $129.8( \pm 15.0)$ & $122.5( \pm 14.1)$ \\
\hline Pepsinogen II/ $\mu \mathrm{g} / \mathrm{l}( \pm \mathrm{CI})$ & $30.3( \pm 13.5)$ & $24.6( \pm 3.4)$ & $25.2( \pm 3.3)$ \\
\hline Pepsinogen I/II $( \pm \mathrm{CI})$ & $2.0( \pm 0.3)$ & $8.8( \pm 4.7)$ & $8.1( \pm 4.7)$ \\
\hline Gastrin-17 pmol/1 $( \pm \mathrm{CI})$ & $2.3( \pm 2.0)$ & $2.2( \pm 1.8)$ & $2.2( \pm 1.7)$ \\
\hline \multicolumn{4}{|l|}{ Hp IgG ELISA } \\
\hline Prevalence (\%) & $14 / 15(93.3)$ & $59 / 71(83.1)$ & $73 / 86(84.9)$ \\
\hline Mean $( \pm \mathrm{CI})$ positive samples $(\geq 30$ EIU) & $303.6( \pm 189.6)$ & $247.6( \pm 69.1)$ & $251.5( \pm 64.5)$ \\
\hline Histology & $5 / 7(71.4)$ & $65 / 79(82.3)$ & $70 / 86(81.4)$ \\
\hline H. pylori prevalence $(\%)$ & $0 / 14$ & $14 / 72$ & $14(16.3 \%)$ \\
\hline Normal gastric mucosa & $8 / 14$ & $57 / 72$ & $65(75.7 \%)$ \\
\hline Superficial ( non atrophic gastritis) & $1 / 14$ & $0 / 72$ & $1(1.2 \%)$ \\
\hline Atrophic gastritis in corpus & $3 / 14$ & $1 / 72$ & $4(4.7 \%)$ \\
\hline Atrophic gastritis in antrum & $2 / 14$ & $0 / 72$ & $2(2.3 \%)$ \\
\hline Atrophic gastritis in antrum and corpus & & & \\
\hline
\end{tabular}

PGI: Pepsinogen I; PG II: Pepsinogen II; G-17: Gastrin-17 fasting levels; HpIgG: Helicobacter pylori immunoglobulin. 
Table 2. Correlation between diagnosis obtained with histology and those obtained with the blood test panel.

\begin{tabular}{|c|c|c|c|c|c|}
\hline & & \multicolumn{3}{|c|}{ HISTOLOGY } & \multirow{2}{*}{ Tota } \\
\hline & & Normal mucosa & Non atrophic gastritis & Atrophic gastritis & \\
\hline \multirow{3}{*}{ GASTRO BLOOD TEST PANEL } & Normal mucosa & 4 & 6 & 0 & 10 \\
\hline & Non atrophic gastritis & 10 & 51 & 1 & 62 \\
\hline & Atrophic gastritis & 0 & 9 & 6 & 14 \\
\hline Total & & 14 & 65 & 7 & 86 \\
\hline
\end{tabular}

NB: Atrophic gastritis included: Atrophic gastritis in the antrum, Atrophic gastritis in the corpus and Atrophic gastritis in both antrum and corpus; non atrophic gastritis $=$ superficial gatristis; No gastritis $=$ Normal gastric mucosa.

\section{DISCUSSION}

H. pylori infection is one of the most common chronic infections in the majority of the global population [9]. In most $H$. pylori infected cases, gastritis progress over years into atrophic type, which considerably increase the risk of gastric adenoma, cancer and MALT lymphoma [10]. Approximately $10 \%$ of patients suffering from gastritis caused by $H$. pylori develop severe Atrophic gastritis of the corpus $[11,12]$. An early diagnosis of atrophic gastritis and the eradication of $H$. pylori form bases for treatment of atrophic gastritis and the prevention of related diseases. The prevalence of $H$. pylori infection obtained $(81.40 \%)$ and that for atrophic gastritis $(8.1 \%)$. The finding is consistent with the reports that atrophic gastritis prevalence in the world is about $10 \%[11,12]$. In the diagnosis of $H$. pylori infection by the GastroPanel test the sensitivity and specificity were $95.7 \%$ and $62.5 \%$ respectively, with $91.8 \%$ of PPV and $76.9 \%$ of NPV. Although the serological pattern of $H$. pylori infection was statistically similar to the histological pattern $(\mathrm{p}>0.50)$ (Table 1), the low specificity and NPV are however, due to levels of H. pylori $\operatorname{IgG}$ which can remain elevated for up to six to twelve months even after eradication and thus not being able to distinguish between past and recent infection [1]. But in conjunction with pepsinogens and gastrin-17 it can serve to diagnose inflammation related to other causes such as autoimmunity and use of nonsteroidal anti inflammatory drugs $[1,4]$.

A statististically significant decrease in the mean PGI concentrations with increasing atrophic stages in the corpus was observed $(p=0.003)$. These findings were consistent with the report of Pasechnikov et al. (2005) [10] that serum pepsinogens decrease as atrophic gastritis in the corpus worsens due to loss of mucosal glands and cells. The use of serum pepsinogen I levels as an assessment of gastric acid secretion was adopted as early as in $1985[13,14]$. The clinical significance of pepsinogen A and pepsinogen $\mathrm{C}$ and serum gastrin levels [15] and the role of serum pepsinogen I and serum gastrin in the screening of severe atrophic corpus gastritis had been studied [16] and in screening of atrophic pan gastritis with high risk of cancer [17]. PGI reflects the status of the mucosa of the corpus and fundus of the stomach and is a wellknown indicator of the corpus mucosa.

For the scoring of atrophy by the GastroPanel test the sensitivity, specificity, PPV and NPV were respectively $85.7 \%, 88.6 \%, 40.0 \%$ and $98.6 \%$. These results are similar with what has been reported in many other countries for example Sipponen et al. (2002) [18] in Finland; Väänänen et al. (2003) [19] in Finland; Pasechnikov et al. (2004) [20] in Russia; Cavallaro et al. (2004) [21] in Italy respectively reported the accuracy, sensitivity and specificity of the GastroPanel test as (91\%:89\%:93:\%); (81\%:79\%:91\%); (84\%:79\%:96\%) and (96\%:78\%:98\%). There was however, a significant difference in the diagnosis of atrophic antrum gastritis between the GastroPanel and histology $(\mathrm{p}<0.01)$. This difference also looking at the low PPV value may be due to false positive diagnosis of atrophic antrum gastritis. We used fasting gastrin levels which can not differentiate between atrophic antrum gastritis and high intragastric acid output. According to Di Mario et al. 2008 [1] and Sipponen et al. 2002 [19] high acid secretion may inhibit the release of G-17 from antral G-cells, resulting in low serum levels of G-17 and in false interpretation of the presence of antral atrophy. However, these may be the patients in whom the risk of acid -related duodenogastric or gastro-oesophageal diseases is highest. Differentiation between high acid output and atrophic antrum gastritis can be achieved with the measurement of stimulated gastrin after a protein drink [4]. None the less significant reduced levels of gastrin- 17 $(\mathrm{p}=0.037)$ with progression to antrum atrophy was observed implicating its role in atrum atrophy diagnosis.

\section{CONCLUSION}

The diagnosis of atrophic gastritis obtained with the blood test panel of G-17, PGI and H. pylori antibodies is in a strong agreement with the biopsy findings, and thus the GastroPanel test can be a useful non endoscopic assessment of stomach mucosal atrophy in patients with dyspepsia. Due to its high sensitivity for atrophic gastritis diagnosis, it can be used in the screening of patients at risk of gastric cancer and peptic ulcer disease. Similarly, because of its high specificity for detecting normal stom- 
ach mucosa and non atrophic gastritis (superficial gastritis), it can be used to avoid endoscopic examination. The latter of which is invasive, expensive and limited.

\section{ACKNOWLEDGEMENTS}

Special thanks to Dr. AWONO Parfait of OCEAC Cameroon.

\section{REFERENCES}

[1] Di Mario, F., Franzè, A. and Cavallaro, L.G. (2008) Non-invasive approach to diagnosis of upper gastrointestinal diseases.

[2] Kokkola, A., Rautelin, H. and Puolakkainen, P. (1998) Positive result in serology indicates active Helicobacter pylori infection in patients with atrophic gastritis. Journal of Clinical Microbiology, 36, 1808-1810.

[3] Borody, T.J., Andrews, P., Jankiewicz, E., et al. (1993) Apparent reversal of early gastric mucosal atrophy after triple therapy for Helicobacter pylori. American Journal of Gastroenterology, 88, 1266-1268.

[4] www.biohit.com/diagnostics-servicelaboratory/March2011

[5] www.biohit.com/gastropanel-gastropanel research applications/March 2011

[6] http://www.gastropanel.net/ http://www.biohit.com/Diagnostics/Litterature/March2011

[7] Dixon, M.F., Genta, R.M. and Yardley, J.H. (1996) Classification and grading of gastritis. The updated Sydney system. American Journal of Pathology, 20, 1161-1181. doi:10.1097/00000478-199610000-00001

[8] Burtis, C.A. and Ashwood E.R. (1999) Text Book of Clinical Chemistry. 3rd Edition, 310-319.

[9] Sipponen, P., Harkonen, M. and Arto, A. (2001) Determination of atrophic gastritis from serum sample. Finnish Medicine Journal, 38, 3833-3839.

[10] Pasechnikov, V.D., Sergey, C.Z., Sergey, M., et al. (2005) Invasive and non invasive diagnosis of Helicobacter pylori-associated atrophic gastritis: A comparative study. Scandinavian Journal of Gastroenterology, 40, 297-301. doi:10.1080/00365520410010607

[11] Varis, K., Sipponen, P., Laxén, F., Samloff, M., Huttunen, J.K., Taylor, P.R., Heinonen, O.P., Albanes, D., Sande, N., Virtamo, J., Härkönen, M. and the Helsinki Gastritis Study Group (2000) Implications of serum pepsinogen I in early endoscopic diagnosis of gastric cancer and dysplasia. Scandinavian Journal of Gastroenterology, 35, 950-956. doi:10.1080/003655200750023011

[12] Suovaniemi, O., Harkonen, M., Paloheimo, L. and Sipponen, P. (2003) GastroPanel: Diagnosing atrophic gas- tritis from serum providing a tool for evidence-based medicine. Business Briefing Global Health Care, 1-4. www.biohit.com

[13] Goedhard, J.G., Biemond, I., Gilians, J.P. and Pals, G. (1985) Serum pepsinogen I levels: Assessment of gastric acid secretion. Progress Clinical and Biological Research, 173, 139-146.

[14] Massarrat S. (1985) Serum pepsin activity as a parameter of gastric acid secretion. Hepato-Gastroenterology, 32, 185-190.

[15] Westerveld, B.D., Pals, G., Lamers, C.B., Defize, J., Pronk, J.C., Frants, R.R., Ooms, E.C., Kreuning, J., Kostense, P.J. and Eriksson, A.W. (1987) Clinical signifycance of pepsinogen A isozymogens, serum pepsinogen $\mathrm{A}$ and $\mathrm{C}$ levels, and serum gastrin levels. Cancer, 59, 952-958.

doi:10.1002/1097-0142(19870301)59:5<952::AID-CNC $\underline{\mathrm{R} 2820590517>3.0 . \mathrm{CO} ; 2-\mathrm{G}}$

[16] Kekki, M., Samloff, I.M., Varis, K. and Ihamaki, T. (1991) Serum pepsinogen I and gastrin-17 in the screening of severe atrophic corpus gastritis. Finland. Scandinavian Journal of Gastroenterology Supplement, 186, 109-116. doi:10.3109/00365529109103997

[17] Varis, K., Kekki, M., Härkönen, M., Sipponen, P. and Samloff, I.M. (1991). Serum pepsinogen I and serum gastrin in the screening of atrophic pangastritis with high risk of gastric cancer. Scandinavian Journal of Gastroenterology, 186, 117-123. doi:10.3109/00365529109103998

[18] Sipponen, P, Suovaniemi, O, Harkonen, M. and Paloheimo, L.I. (2002) Diagnosis of atrophic gastritis from a serum sample. Jordan Medical Journal, 36, 117-121.

[19] Väänänen, H., Vauhkonne, M., Helske, T., Kääriänen, I., Rasmussen, M., Tunturi, H., Koskenpato, J., Sotka, M., Turunen, M., Sandströmme, R., Ristikankare, M., Jussila, A. and Sipponen, P. (2003) Non endoscopic diagnosis of atrophic gastritis with a blood test. Correlation between gastric histology and serum levels of gastrin-17 and pepsinogen 1: A multicenter study. European Journal of Gastroenterology and Hepatology, 15, 885-891. doi:10.1097/00042737-200308000-00009

[20] Pasechnikov, V.D., Chukov, S.Z., Kotelevets, S.M., Mostovov, A.N., Mernova, V.P. and Polyakova, M.B. (2004) Possibilityof non-invassive diagnosis of gastric mucosal precancerous changes. World Journal of Gastroenterology, 10, 3146-3150.

[21] Cavallaro L.G, Moussa A.M, Liatoupou S., Comparato G., Bertolini S., Merli R., et al. (2004) Accuracy of "serogical gastric biopsy" in a cohort of dyspeptic patients. Emer- gencies in Gastroenterology, 6th European Bridging Meeting in Gastroenterology, Kaunas, 32. 